\title{
Resource Allocation for Cooperative Transmission in Wireless Networks with Orthogonal Users
}

\author{
D. Richard Brown III \\ Electrical and Computer Engineering Department \\ Worcester Polytechnic Institute \\ Worcester, MA 01609 \\ email: drb@wpi.edu
}

\begin{abstract}
This paper investigates the problem of efficient power allocation in a wireless communication system with two cooperating sources and one destination. The sources in the system each transmit information to a single destination at a fixed SNR target and cooperate via an orthogonal amplify-andforward protocol with two timeslots. We develop a framework for power allocation in this scenario around the concept of "cooperation ratios" and derive expressions for the transmit power required by each source to achieve their SNR targets as a function of these cooperation ratios. Numerical examples are presented for time-invariant channels as well as Rayleigh fading channels. Our results show that cooperation does not reduce required transmit powers when both sources have symmetric time-invariant channels to the destination. When sources have asymmetric time-invariant channels to the destination, total power is minimized when only the source with the stronger channel cooperates. In the case of Rayleigh fading channels, we demonstrate that mutual cooperation can minimize the average total required transmit power and can also lead to a reduction in average required transmit power for both sources.
\end{abstract}

\section{INTRODUCTION}

Recently, researchers have recognized that spatial diversity can be achieved in multiuser communication systems even if the nodes in the system each have only one antenna. Sendonaris, Erkip, and Aazhang were the first to propose the concept of user cooperation diversity where nearby users in a cellular system form cooperative "partnerships" by sharing their antennas to achieve increased rate or decreased outage probability in the uplink [1]. Since this seminal work, there has been a growing interest in developing cooperative transmission protocols and understanding the performance limits of user cooperation diversity, c.f. [2]-[6].

In addition to its demonstrated potential for increased rate or decreased outage probability, the user cooperation diversity can also potentially reduce the transmit power required by nodes to meet QoS targets and, consequently, extend the battery life of cooperating nodes [1]. This may be particularly important in energy-constrained scenarios such as sensor networks. Cooperative transmission is unique, however, in that it requires autonomous nodes to allocate transmit power between selfish and cooperative transmissions. Inefficient allocation of transmit power could lead to worse power efficiency than no cooperation. The primary focus of this paper is on the problem of how to allocate transmit power between selfish and cooperative transmissions in order to maximize power efficiency in a wireless communication system with two cooperating nodes communicating independent information over orthogonal subchannels to one destination. Our analysis also considers the problem of fairness: Under what conditions do both nodes benefit (in terms of reduced transmit power) from cooperation? This question may be important towards developing a better understanding of the problem of inducing cooperation between autonomous nodes.

\section{System Model ANd CoOperative Protocol}

The two-source cooperative transmission system model considered in this paper is shown in Figure 1. The channels are assumed to be flat and either time-invariant or block-fading where their value is randomly generated but remains constant over both timeslots of the cooperative protocol described below. The channels are also assumed to be known to both sources as well as the destination.

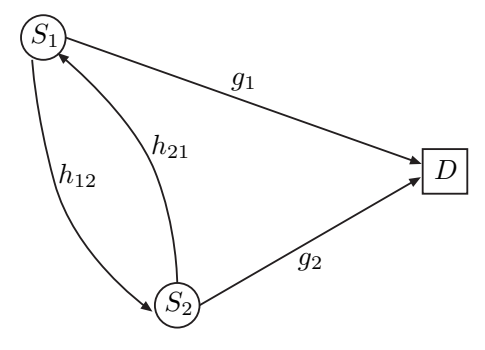

Fig. 1. Two-source cooperative transmission system model.

The two-source amplify-and-forward [7] cooperative transmission protocol is given in Table I. Transmission occurs in two timeslots: each source transmits its own information in the first timeslot; the second timeslot is used for cooperative retransmission. We denote the $i^{\text {th }}$ source's zero-mean unitvariance information symbol as $x_{i}$, the $i^{\text {th }}$ source's amplitude in the $k^{\text {th }}$ timeslot as $a_{i}[k] \geq 0$, and the $i^{\text {th }}$ source's transmission in the $k^{\text {th }}$ timeslot as $t_{i}[k]$. The $j^{\text {th }}$ source in the system receives the signal sent by the $i^{\text {th }}$ source in timeslot $k$ as

$$
r_{i j}[k]=h_{i j} t_{i}[k]+v_{i j}[k]
$$

where $h_{i j}$ is the (scalar) channel gain in orthogonal channel $i$ between source $i$ and source $j$ and $v_{i j}[k]$ is the zero-mean noise in this channel with variance $\sigma_{v}^{2}>0$. 
TABLE I

TWO-SOURCE AMPLIFY-AND-FORWARD COOPERATIVE PROTOCOL.

\begin{tabular}{|l|l|l|}
\hline & timeslot 1 & timeslot 2 \\
\hline \hline Source 1 & $t_{1}[1]=a_{1}[1] x_{1}$ & $t_{1}[2]=a_{1}[2] r_{21}[1]$ \\
\hline Source 2 & $t_{2}[1]=a_{2}[1] x_{2}$ & $t_{2}[2]=a_{2}[2] r_{12}[1]$ \\
\hline
\end{tabular}

The signal received by the destination in the $i^{\text {th }}$ orthogonal channel in timeslot $k$ is given as

$$
y_{i}[k]=g_{i} t_{i}[k]+w_{i}[k]
$$

where $g_{i}$ is the scalar channel gain between the $i^{\text {th }}$ user and the destination and $w_{i}[k]$ is the zero-mean noise in this channel with variance $\sigma_{w}^{2}>0$. The destination forms the decision statistic for the $i^{\text {th }}$ user's information symbol as a linear combination of the two relevant observations, i.e.,

$$
\begin{aligned}
& y_{1}=b_{1}[1] y_{1}[1]+b_{2}[2] y_{2}[2] \\
& y_{2}=b_{2}[1] y_{2}[1]+b_{1}[2] y_{1}[2]
\end{aligned}
$$

where $b_{i}[k]$ are the linear combination parameters selected by the destination to maximize the SNR of the decision statistics. Note that there is no multiaccess interference due to the orthogonality of all transmissions in this protocol.

\section{TRANSmit POWER AND SNR ANALYSIS}

Given the system model and cooperative transmit protocol described in Section II, we consider the problem of efficiently allocating transmit power in order to achieve a pair of fixed SNR targets, denoted as $\mathrm{SNR}_{1}$ and $\mathrm{SNR}_{2}$, at the destination. In the absence of cooperation, the orthogonality of the sources makes the solution to this problem straightforward. The SNR targets will be satisfied iff $P_{i}=a_{i}^{2}[1] \geq\left(\sigma_{w}^{2} / g_{i}^{2}\right) \mathrm{SNR}_{i}$ where $P_{i}$ denotes the transmit power of the $i^{\text {th }}$ source. The problem of power allocation in the cooperative scenario is less straightforward, however, due to the fact that there are four transmit powers to specify with only two constraints. This section develops an analytical framework for power allocation in the two-source cooperative transmission scenario in order to better understand how cooperation influences the individual power requirements of each source as well as the total power requirement for both sources.

\section{A. Transmit Powers and Cooperation Ratios}

The first step in our analysis is to calculate the transmit power for each source in the original and cooperative timeslots. Denoting the transmit power of source $i$ in timeslot $k$ as $P_{i}[k]:=\mathrm{E}\left[t_{i}^{2}[k]\right]$, the transmit powers in each timeslot (conditioned on the channel realizations) are given explicitly in Table II. The total transmit power for source $i$ is given as $P_{i}=P_{i}[1]+P_{i}[2]$ and the total transmit power over all sources is given as $P_{\text {tot }}=P_{1}+P_{2}$.

From the power expressions in Table II, we can define a "cooperation ratio" parameter for each source in the system. The $i^{\text {th }}$ source's cooperation ratio is defined as the ratio of
TABLE II

TRANSMIT POWERS BY SOURCE AND TIMESLOT.

\begin{tabular}{|l|l|l|}
\hline & timeslot 1 & timeslot 2 \\
\hline \hline Source 1 & $P_{1}[1]=a_{1}^{2}[1]$ & $P_{1}[2]=a_{1}^{2}[2]\left(h_{21}^{2} a_{2}^{2}[1]+\sigma_{v}^{2}\right)$ \\
\hline Source 2 & $P_{2}[1]=a_{2}^{2}[1]$ & $P_{2}[2]=a_{2}^{2}[2]\left(h_{12}^{2} a_{1}^{2}[1]+\sigma_{v}^{2}\right)$ \\
\hline
\end{tabular}

the power of the $i^{\text {th }}$ source's cooperative retransmission to the power of the original transmission of source $j(i \neq j)$. Using the results from Table II, we can write the cooperation ratios for the $i^{\text {th }}$ source as

$$
\alpha_{i}:=\frac{P_{i}[2]}{P_{j}[1]}=\frac{a_{i}^{2}[2]\left(h_{j i}^{2} a_{j}^{2}[1]+\sigma_{v}^{2}\right)}{a_{j}^{2}[1]} \quad i, j \in\{1,2\}
$$

for $0 \leq \alpha_{i}<\infty$ and $j \neq i$. We note that the noncooperative case corresponds to $\alpha_{1}=\alpha_{2}=0$.

\section{B. Destination Processing and SNR}

The next step in our analysis is to derive expressions for the SNR of $y_{1}$ and $y_{2}$ at the destination under the assumption that the destination optimally combines the observations $\left\{y_{1}[1], y_{1}[2], y_{2}[1], y_{2}[2]\right\}$ to maximize SNR. Assuming that all of the noise terms in the observations are mutually independent as well as independent of the data, it can be shown that maximal ratio combining (MRC) at the destination maximizes the SNR of $y_{1}$ and $y_{2}$. The MRC combining coefficients can be written as

$$
\begin{aligned}
& \frac{b_{2}[2]}{b_{1}[1]}=\frac{g_{2} h_{12} a_{2}[2] \sigma_{w}^{2}}{g_{1}\left(\sigma_{w}^{2}+g_{2}^{2} a_{2}^{2}[2] \sigma_{v}^{2}\right)} \\
& \frac{b_{1}[2]}{b_{2}[1]}=\frac{g_{1} h_{21} a_{1}[2] \sigma_{w}^{2}}{g_{2}\left(\sigma_{w}^{2}+g_{1}^{2} a_{1}^{2}[2] \sigma_{v}^{2}\right)}
\end{aligned}
$$

and the resulting SNRs with MRC at the destination can be expressed as

$$
\begin{aligned}
& \mathrm{SNR}_{1}=\frac{g_{1}^{2} a_{1}^{2}[1]}{\sigma_{w}^{2}}+\frac{g_{2}^{2} a_{2}^{2}[2] h_{12}^{2} a_{1}^{2}[1]}{\sigma_{w}^{2}+g_{2}^{2} a_{2}^{2}[2] \sigma_{v}^{2}} \\
& \mathrm{SNR}_{2}=\frac{g_{2}^{2} a_{2}^{2}[1]}{\sigma_{w}^{2}}+\frac{g_{1}^{2} a_{1}^{2}[2] h_{21}^{2} a_{2}^{2}[1]}{\sigma_{w}^{2}+g_{1}^{2} a_{1}^{2}[2] \sigma_{v}^{2}} .
\end{aligned}
$$

Substituting the cooperation ratios and the transmit powers from Table II into these expressions, we can rewrite the SNR of each source as

$$
\begin{aligned}
\mathrm{SNR}_{1} & =\frac{g_{1}^{2} P_{1}[1]}{\sigma_{w}^{2}}+\frac{\alpha_{2} P_{1}^{2}[1] g_{2}^{2} h_{12}^{2}}{\sigma_{w}^{2}\left(h_{12}^{2} P_{1}[1]+\sigma_{v}^{2}\right)+\alpha_{2} P_{1}[1] g_{2}^{2} \sigma_{v}^{2}} \\
\mathrm{SNR}_{2} & =\frac{g_{2}^{2} P_{2}[1]}{\sigma_{w}^{2}}+\frac{\alpha_{1} P_{2}^{2}[1] g_{1}^{2} h_{21}^{2}}{\sigma_{w}^{2}\left(h_{21}^{2} P_{2}[1]+\sigma_{v}^{2}\right)+\alpha_{1} P_{2}[1] g_{1}^{2} \sigma_{v}^{2}} .
\end{aligned}
$$

We note that specification of the SNR targets $\left\{\mathrm{SNR}_{1}, \mathrm{SNR}_{2}\right\}$ as well as the cooperation ratios $\left\{\alpha_{1}, \alpha_{2}\right\}$ fully determines the minimum transmit powers for both sources in first timeslot and the resulting cooperative transmit powers in the second timeslot. 


\section{Results}

This section uses the analysis of Section III to examine the transmit power required by each source as well as the total transmit power required to satisfy a pair of SNR targets SNR 1 and $\mathrm{SNR}_{2}$ at a fixed level of cooperation specified by $\alpha_{1}$ and $\alpha_{2}$. We consider two scenarios distinguished by the channel model: time-invariant channels and Rayleigh fading channels. Numerical examples are presented in order to develop insight into the following questions:

1) What choice of cooperation ratios $\alpha_{1}$ and $\alpha_{2}$ minimize the total required transmit power $P_{t o t}$ ?

2) How can we describe the set of mutually beneficial cooperation ratios $\mathcal{A}=\left\{0<\alpha_{1}, \alpha_{2}<\infty \mid P_{i}\left(\alpha_{1}, \alpha_{2}\right)<\right.$ $\left.P_{i}(0,0) \forall i \in\{1,2\}\right\}$ ? Under what conditions is this set empty or non-empty?

All numerical results in this section have fixed SNR targets specified as $\mathrm{SNR}_{1}=\mathrm{SNR}_{2}=10 \mathrm{~dB}$.

\section{A. Time-Invariant Channels}

In this section, we examine the transmit power requirements for the case when all of the channels in Figure 1 are all modeled as time-invariant. We first consider the case where $g_{1}=g_{2}$ and $h_{12}=h_{21}$, i.e., symmetric channels. In this case, it can be shown that cooperative transmission can result in reduced individual transmit power for one source at the expense of increased transmit power for the other source. A simultaneous reduction in individual transmit powers $P_{1}$ and $P_{2}$ with respect to the noncooperative case is not possible, however, hence $\mathcal{A}=\emptyset$. Moreover, the total required transmit power is minimized when $\alpha_{1}=\alpha_{2}=0$. In other words, cooperation cannot provide a reduction in total transmit power with respect to the noncooperative case. These results can be intuitively explained by the fact that, if all of the channels are symmetric, it is more effective to put power into the the first timeslot than to amplify and forward the noisy signal from the other source in the second timeslot. Figure 2 shows the required transmit powers $P_{1}, P_{2}$, and $P_{t o t}$ as a function of the cooperation ratios $\alpha_{1}$ and $\alpha_{2}$ for a particular example of the symmetric time-invariant channel case.

A more interesting case occurs when the source-destination channels $g_{1}$ and $g_{2}$ are asymmetric. In this case, it can be shown that, like the symmetric case, cooperative transmission can reduce the individual transmit power for one source at the expense of increased transmit power for the other source. A simultaneous reduction in individual transmit powers is not possible and, again, $\mathcal{A}=\emptyset$. Unlike the symmetric case, however, as long as the source-source channel is better than the weaker source-destination channel, cooperation can reduce the total required transmit power with respect to the noncooperative case when the source with the stronger sourcedestination channel cooperates. In this sense, mutual cooperation is undesirable: only the source with the stronger channel to the destination should retransmit in the second timeslot. Figure 3 shows a particular example of the time-invariant asymmetric channel case when $g_{1}<g_{2}$ and $h_{12}=h_{21}$.

\section{B. Fading Channels}

In this section, we analyze the average transmit power required to meet the fixed SNR targets $\left\{\mathrm{SNR}_{1}, \mathrm{SNR}_{2}\right\}$ for a fixed set of cooperation ratios $\left\{\alpha_{1}, \alpha_{2}\right\}$ in the case when all of the channels in Figure 1 are modeled as flat independent Rayleigh fading. In this case, the sources allocate power according to the current channel state in order to meet their SNR targets. We assume that there is no maximum power constraint on either source in this analysis.

In order to quantify the benefits of cooperation, we first consider the problem of meeting a fixed SNR target in the noncooperative case with Rayleigh fading channels. In this case, all power is allocated to timeslot 1 and the $i^{\text {th }}$ source's required transmit power, conditioned on the channel realization, is $P_{i}=\left(\sigma_{w}^{2} / g_{i}^{2}\right) \operatorname{SNR}_{i}$. When $g_{i}$ is modeled as a Rayeligh distributed random variable, it can be shown that $g_{i}^{2}$ is exponentially distributed and that the average transmit power $\mathrm{E}\left[P_{i}\right]$ is infinite irrespective of the mean of $g_{i}$.

Proposal 1: Given $g_{1}, g_{2}, h_{12}$, and $h_{21}$ are independent and Rayleigh distributed, and $\alpha_{1}, \alpha_{2} \in(0, \infty)$, the average transmit powers $\mathrm{E}\left[P_{1}\right]$ and $\mathrm{E}\left[P_{2}\right]$ required to meet the fixed SNR targets $\mathrm{SNR}_{1}<\infty$ and $\mathrm{SNR}_{2}<\infty$ are finite.

Proof sketch: Due to space limitations, a sketch of the proof to Proposition 1 is provided here. The average total transmit power by the $i^{\text {th }}$ source can be expressed as $\mathrm{E}\left[P_{i}\right]=\mathrm{E}\left[P_{i}[1]\right]+$ $\mathrm{E}\left[P_{i}[2]\right]$. By definition of the cooperation ratio $\alpha_{i}$, we can state that $\mathrm{E}\left[P_{i}[2]\right]<\infty$ if $\alpha_{i}$ is finite and $\mathrm{E}\left[P_{j}[1]\right]<\infty$ for $j \neq i$. Hence, given the assumption that the cooperation ratios are finite, each source's total average required transmit power is finite if $\mathrm{E}\left[P_{1}[1]\right]<\infty$ and $\mathrm{E}\left[P_{2}[1]\right]<\infty$.

Isolating the timeslot 1 transmit power for the $i^{\text {th }}$ source, we can rewrite (1) as

$P_{i}[1]=a_{i}^{2}[1]=\operatorname{SNR}_{i} \frac{\sigma_{w}^{4}+a_{j}^{2}[2] \sigma_{w}^{2} \sigma_{v}^{2} Y}{\sigma_{w}^{2} X+a_{j}^{2}[2] \sigma_{v}^{2} X Y+a_{j}^{2}[2] \sigma_{w}^{2} Y Z}$

where we have substituted $X=g_{i}^{2}, Y=g_{j}^{2}$, and $Z=h_{i j}^{2}$ for the random independent exponentially distributed squaredchannel coefficients. For notational convenience, we normalize the means of $X, Y$, and $Z$ and collect the non-random parameters to write

$$
P_{i}[1]=\frac{\theta_{0}+\theta_{1} Y}{\theta_{2} X+\theta_{3} X Y+\theta_{4} Y Z}=P_{i 0}[1]+P_{i 1}[1]
$$

where $0<\theta_{i}<\infty$ for all $i \in\{0, \ldots, 4\}$ and $P_{i 0}[1]$ and $P_{i 1}[1]$ correspond to the fraction formed by the first and second terms of the numerator, respectively. To show that $\mathrm{E}\left[P_{i}[1]\right]$ is finite, we will show separately that $\mathrm{E}\left[P_{i 0}[1]\right]<\infty$ and $\mathrm{E}\left[P_{i 1}[1]\right]<\infty$.

We can upper bound $P_{i 1}[1]$ by

$P_{i 1}[1]=\frac{\theta_{1} Y}{\theta_{2} X+\theta_{3} X Y+\theta_{4} Y Z} \leq \frac{\theta_{1}}{\theta_{3} X+\theta_{4} Z} \leq \frac{c}{X+Z}$

for some finite constant $c$ where we have used the fact that $\theta_{2} X \geq 0$. An upper bound on the expectation of $P_{i 1}[1]$ can then be written as

$$
\mathrm{E}\left[P_{i 1}[1]\right] \leq \int_{0}^{\infty} \int_{0}^{\infty} \frac{c}{x+z} e^{-x} e^{-z} d x d z
$$


which can be shown to be finite by using the bounds $e^{-x} \leq$ $(1+x)^{-1}$ and $e^{-y} \leq(1+y)^{-1}$ for all $x, y \geq 0$.

Following a similar procedure for $P_{i 0}[1]$, we can write an upper bound

$P_{i 0}[1]=\frac{\theta_{0}}{\theta_{2} X+\theta_{3} X Y+\theta_{4} Y Z} \leq \frac{\theta_{0}}{\theta_{2} X+\theta_{4} Y Z} \leq \frac{d}{X+Y Z}$

for some finite constant $d$ where we have used the fact that $\theta_{3} X Y \geq 0$. An upper bound on the expectation of $P_{i 0}[1]$ can then be written as

$$
\mathrm{E}\left[P_{i 0}[1]\right] \leq \int_{0}^{\infty} \int_{0}^{\infty} \int_{0}^{\infty} \frac{d}{x+y z} e^{-x} e^{-y} e^{-z} d x d y d z .
$$

We can use the continuity of the integrand and the monotonicity of the exponential terms to write a looser upper bound on this expectation as

$\mathrm{E}\left[P_{i 0}[1]\right] \leq \sum_{j=0}^{\infty} \sum_{k=0}^{\infty} \sum_{\ell=0}^{\infty} e^{-j} e^{-k} e^{-\ell} \int_{j}^{j+1} \int_{k}^{k+1} \int_{\ell}^{\ell+1} \frac{d}{x+y z} d x d y d z$.

It can be shown that $\int_{0}^{1} \int_{0}^{1} \int_{0}^{1} \frac{d}{x+y z} d x d y d z$ is finite and, consequently, that $\mathrm{E}\left[P_{i 0}[1]\right]$ is finite. Since both $P_{i 0}[1]$ and $P_{i 1}[1]$ are finite, $P_{i}[1]$ is finite and the total average required transmit power for both sources is finite.

We note, as a technical detail, that the proof of Proposition 1 does not require the source-source channels $h_{12}$ and $h_{21}$ to be independent (which may not be the case if these channels are reciprocal) but only that the sets of channels $\left\{g_{1}, g_{2}, h_{12}\right\}$ and $\left\{g_{1}, g_{2}, h_{21}\right\}$ are independent. Proposition 1 implies that any level of mutual cooperation is beneficial, in the sense of reducing the required average transmit power, when the channels are modeled as independent Rayleigh fading since the average required transmit power in the noncooperative case is infinite for both sources. Hence $\mathcal{A}=(0, \infty) \times(0, \infty)$ in the independent Rayleigh fading channel case.

We now consider two numerical examples to illustrate how cooperation influences the individual power requirements of each source as well as the total power requirement for both sources in the fading channel scenario. We first consider the case where all of the channels are modeled as independent Rayleigh fading and where $\mathrm{E}\left[g_{1}\right]=\mathrm{E}\left[g_{2}\right]$ and $\mathrm{E}\left[h_{12}\right]=$ $\mathrm{E}\left[h_{21}\right]$, i.e., statistically symmetric channels. Figure 4 shows a particular example of this case where, for a fixed choice of cooperation ratios, each source's transmit powers are calculated for each set of channel realizations and these powers are averaged over 1000 iterations. Figure 4 shows that there is an optimal level of mutual cooperation with fixed $\alpha_{1}>0$ and $\alpha_{2}>0$ such that the average total required transmit power is minimized. Figure 5 shows that similar results are obtained in the asymmetric fading case with the primary difference being that contours are skewed such that minimum total power operating point requires the source with the statistically
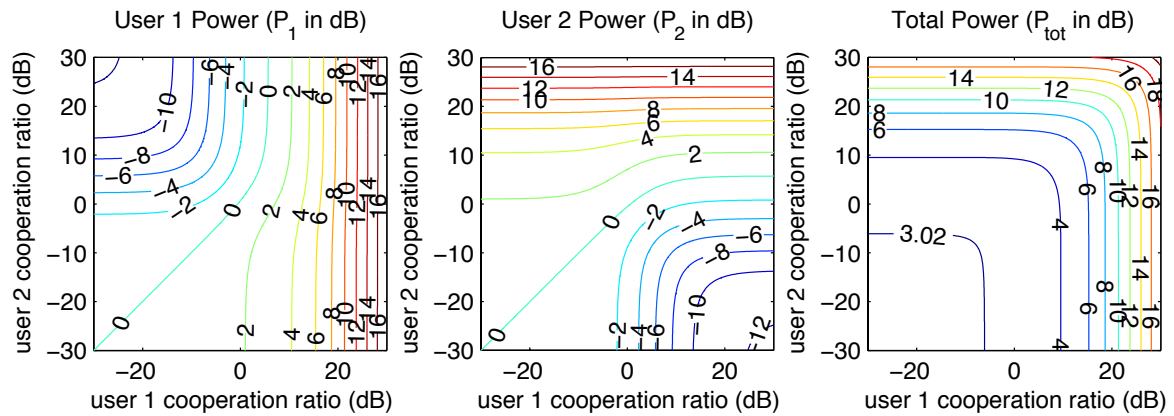

Fig. 2. Two-source cooperative transmission power requirements for the case where $g_{1} / \sigma_{w}=g_{2} / \sigma_{w}=10 \mathrm{~dB}$ and $h_{12} / \sigma_{w}=h_{21} / \sigma_{2}=22 \mathrm{~dB}$. Note that the minimum total transmit power is achieved with no cooperation in this case and there is no choice of $\left\{\alpha_{1}, \alpha_{2}\right\}$ that results in a simultaneous reduction of transmit power for both sources.
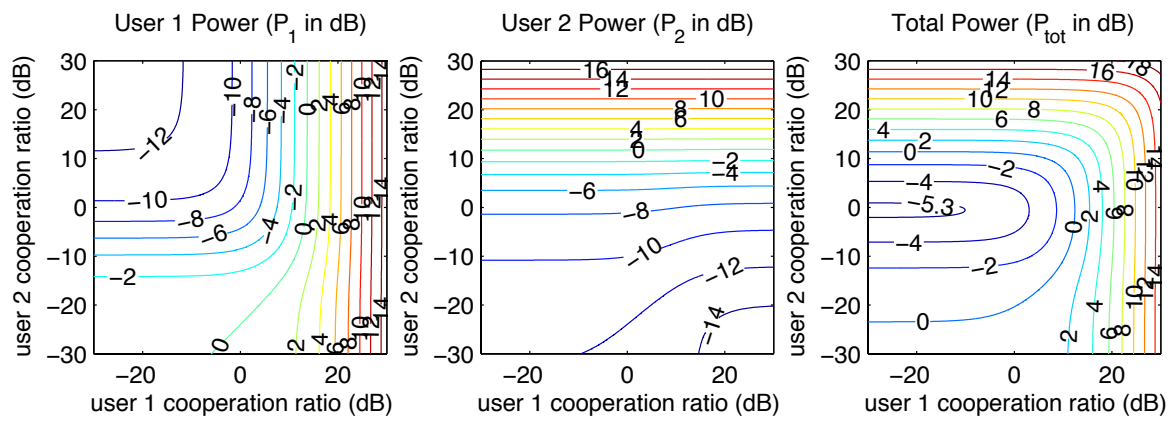

Fig. 3. Two-source cooperative transmission power requirements for the case where $g_{1} / \sigma_{w}=10 \mathrm{~dB}, g_{2} / \sigma_{w}=22 \mathrm{~dB}$ and $h_{12} / \sigma_{w}=h_{21} / \sigma_{2}=22 \mathrm{~dB}$. Note that the minimum total transmit power is achieved when source 2 cooperates $\left(\alpha_{2} \approx 0.9\right)$ and source 1 does not cooperate $\left(\alpha_{1}=0\right)$. Also note that and there is no choice of $\left\{\alpha_{1}, \alpha_{2}\right\}$ that results in a simultaneous reduction of transmit power for both sources. 
stronger channel (source 2, in this case) to have a higher cooperation ratio than the source with the weaker channel. It is beneficial, in terms of average total and individual required powers, for both sources to cooperate even in the asymmetric fading case.

\section{CONCLUSIONS}

This paper considers the problem of efficient power allocation in a wireless communication system with two cooperating sources communicating independent information over orthogonal subchannels to one destination. We developed a framework for power allocation in this scenario around the concept of "cooperation ratios" and derived expressions for the transmit power required by each source to achieve their SNR targets as a function of these cooperation ratios. For the system model and protocol described in Section II, we show that cooperation can reduce the total required transmit power but a simultaneous reduction of the required individual transmit powers $P_{1}$ and $P_{2}$ with respect to the noncooperative case is not possible for any choice of cooperation ratios. This implies that only the source with the stronger channel should cooperate when the channels are time-invariant.

When the channels are independently fading, our results show that cooperation can benefit both sources in terms of reducing their average required individual transmit powers $\mathrm{E}\left[P_{1}\right]$ and $\mathrm{E}\left[P_{2}\right]$ as well as the average total power even when the sources face statistically symmetric channels to the destination. In light of the time-invariant channel results, these results imply that, even though it is instantaneously suboptimal for both sources to cooperate with fixed non-zero cooperation ratios, mutual cooperation is beneficial on average for both sources when the channels are independently fading.

\section{REFERENCES}

[1] A. Sendonaris, E. Erkip, and B. Aazhang, "Increasing uplink capacity via user cooperation diversity," in Proceedings of the 1998 IEEE International Symposium on Information Theory, (Cambridge, MA), p. 156, August 16211998.

[2] J. Laneman and G. Wornell, "Distributed space-time-coded protocols for exploiting cooperative diversity in wireless networks," IEEE Transactions on Information Theory, vol. 49, pp. 2415-2425, October 2003.

[3] A. Stefanov and E. Erkip, "On the performance analysis of cooperative space-time coded systems," in Proceedings of the IEEE Wireless Communications and Networking Conference (WCNC), vol. 2, pp. 729-734, 2003.

[4] K. Azarian, H. El Gamal, and P. Schniter, "On the achievable diversity-vsmultiplexing tradeoff in cooperative channels," in Proceedings of the 38th Annual IEEE Conference on Information Sciences and Systems (CISS), (Princeton, NJ), pp. 956-960, March 17-19 2004.

[5] T. Hunter, S. Sanayei, and A. Nostratinia, "The outage behavior of coded cooperation," in Proceedings of the 2004 IEEE International Symposium on Information Theory, (Chicago, IL), p. 270, June 27-July 22004.

[6] I. Maric and R. Yates, "Cooperative multihop broadcast for wireless networks," IEEE Journal on Selected Areas in Communications, vol. 2004, August accepted to appear in IEEE Journal on Selected Areas in Communications.

[7] J. Laneman, G. Wornell, and D. Tse, "An efficient protocol for realizing cooperative diversity in wireless networks," in Proceedings of the IEEE International Symposium on Information Theory (ISIT), (Washington, DC), p. 294, June 24-29 2001.
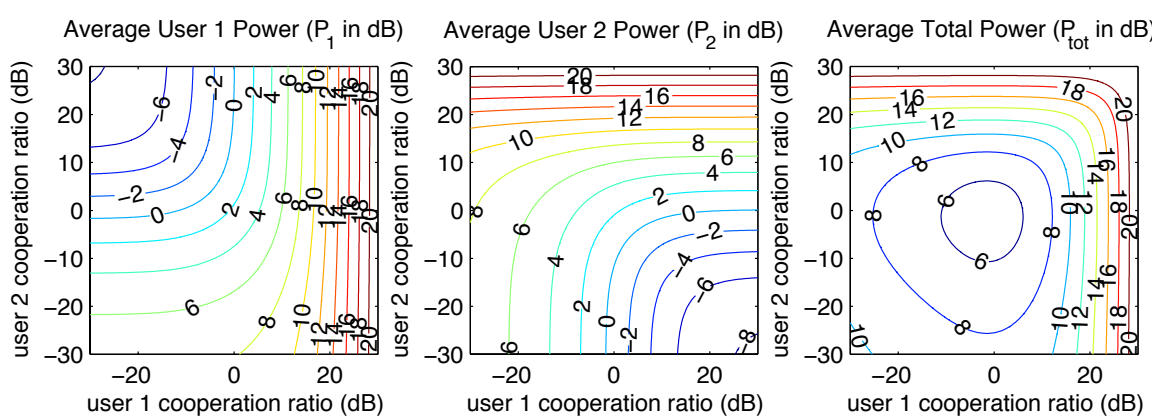

Fig. 4. Two-source cooperative transmission power requirements for the case where all channels are independent and Rayleigh distributed, $\mathrm{E}[\boldsymbol{g}] / \sigma_{w}=$ $\mathrm{E}\left[g_{2}\right] / \sigma_{w}=10 \mathrm{~dB}$ and $\mathrm{E}\left[h_{12}\right] / \sigma_{v}=\mathrm{E}\left[h_{21}\right] / \sigma_{v}=22 \mathrm{~dB}$. The minimum average total transmit power is achieved when $\alpha_{1}=\alpha_{2} \approx 0.7$ in this case.
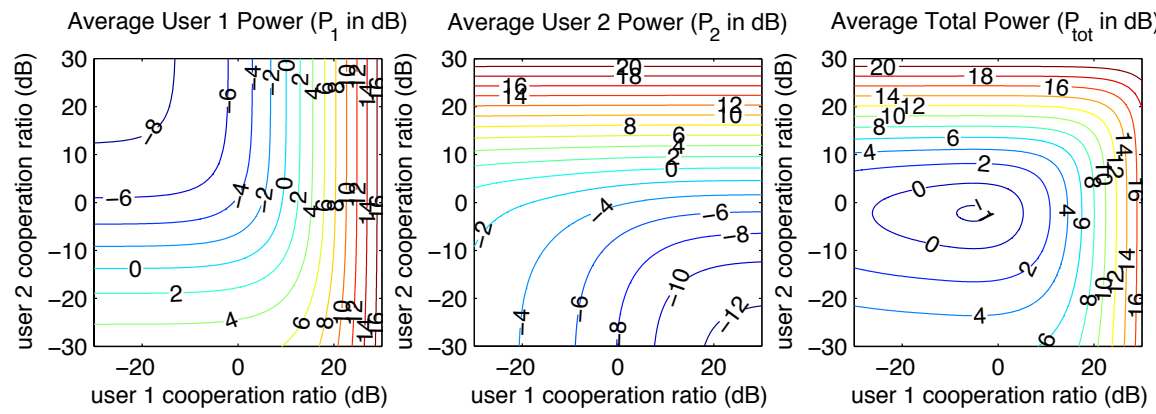

Fig. 5. Two-source cooperative transmission power requirements for the case where all channels are independent and Rayleigh distributed, $\mathrm{E}[g] / \sigma_{w}=10 \mathrm{~dB}$, $\mathrm{E}\left[g_{2}\right] / \sigma_{w}=22 \mathrm{~dB}$, and $\mathrm{E}\left[h_{12}\right] / \sigma_{v}=\mathrm{E}\left[h_{21}\right] / \sigma_{v}=22 \mathrm{~dB}$. The minimum average total transmit power is achieved when $\alpha_{1} \approx 0.32$ and $\alpha_{2} \approx 0.6$ in this case. 